\title{
Collapse of attractive Bose-Einstein condensed vortex states in a cylindrical trap
}

\author{
Sadhan K. Adhikari \\ Instituto de Física Teórica, Universidade Estadual Paulista, 01.405-900 São Paulo, São Paulo, Brazil
}

(Received 29 June 2001; published 18 December 2001)

\begin{abstract}
The quantized vortex states of a weakly interacting Bose-Einstein condensate of atoms with attractive interatomic interaction in an axially symmetric harmonic oscillator trap are investigated using the numerical solution of the time-dependent Gross-Pitaevskii equation obtained by the semi-implicit Crank-Nicholson method. The collapse of the condensate is studied in the presence of deformed traps with the larger frequency along either the radial or the axial direction. The critical number of atoms for collapse is calculated as a function of the vortex quantum number $L$. The critical number increases with increasing angular momentum $L$ of the vortex state but tends to saturate for large $L$.
\end{abstract}

DOI: 10.1103/PhysRevE.65.016703

PACS number(s): 02.70.-c, 02.60.Lj, 03.75.Fi

\section{INTRODUCTION}

Recent experiments [1,2] on Bose-Einstein condensates (BEC's) in dilute bosonic atoms employing magnetic traps at ultralow temperatures have intensified theoretical investigations into various aspects of the condensate [3-7]. The properties of the condensate are usually described by the nonlinear mean-field Gross-Pitaevskii (GP) equation [8], which properly incorporates the trap potential as well as the interaction among the atoms.

Two interesting features of BEC's are (a) the collapse in the case of attractive atomic interaction $[2,7]$ and (b) the possibility of formation of a vortex state in harmonic traps with cylindrical [9-13] as well as spherical [14] symmetry.

For an attractive interatomic interaction [2,7], the condensate is stable for a critical maximum number of atoms. When the number of atoms increases beyond this critical value, due to interatomic attraction the radius of the BEC tends to zero and the maximum density of the condensate tends to infinity. Consequently, the condensate collapses, emitting atoms until the number of atoms is reduced below the critical number and a stable configuration is reached. With a supply of atoms from an external source the condensate can grow again and thus a series of collapses can take place; this has been observed experimentally in the $\mathrm{BEC}$ of ${ }^{7} \mathrm{Li}$ with attractive interaction [2]. Theoretical analyses based on the GP equation also confirm the collapse [7].

The study of superfluid properties of a BEC is of great interest to both theoreticians [9-17] and experimentalists $[18,19]$. The quantized vortex state in a BEC is intimately connected to the existence of superfluidity. Such quantized vortices are expected in superfluid He II. However, due to the strong interaction between helium atoms there is no reliable mean-field description. On the other hand, a weakly interacting trapped BEC is well described by the mean-field GP equation, which is known to admit vortex solutions for a trap with cylindrical symmetry $[9,16]$, and which can be studied numerically. This allows for a controlled theoretical study of quantized vortices in BEC's in contrast to superfluid He II.

Many different techniques for creating vortex states in BEC's have been suggested [12], e.g., stirring the BEC by an external laser at a rate exceeding a critical angular velocity to create a singly quantized vortex line along the axis of rotation [11], spontaneous vortex formation in evaporative cooling [20], controlled excitation to an excited state of atoms [21], and rotation of an axially symmetric trap [22]. Moreover, quantized vortex states in BEC's have been observed experimentally in coupled BEC's comprised of two spin states of ${ }^{87} \mathrm{Rb}$ in a spherical trap, where angular momentum is generated by a controlled excitation of the atoms between the two states [19]. Vortices have also been detected in a single-state $\mathrm{BEC}$ of ${ }^{87} \mathrm{Rb}$ in a cylindrical trap, where angular momentum is generated by a stirring laser beam [18]. Discovery of the possibility of continuously changing the interaction between cold ${ }^{85} \mathrm{Rb}$ atoms by a magnetic-field-induced Feshbach resonance $[23,24]$ suggests that one could experimentally form vortex states in repulsive condensates and study their collapse after transforming them to attractive condensates by such a resonance. Because of the intrinsic interest in BEC's of vortex states in axially symmetric traps, in this work the formation of such a BEC is studied using the numerical solution of the time-dependent GP equation with special attention to its collapse for attractive interatomic interaction.

In general, a vortex line in a nonrotating trapped BEC is expected to be nonstationary. However, it is possible to have dynamically stable vortex BEC states in a nonrotating trap with low quanta of rotational excitation or angular momentum $L$ per particle $[9,15,16,22]$. Vortex BEC states for large repulsive condensates with high quanta of rotational excitation are expected to be unstable and decay to vortices with low quanta $[11,12,14,17]$. In the absence of a vortex, the stable condensate in an axially symmetric trap has a cylindrical shape. Such a BEC has the largest density on the axis of the trap. For a purely attractive interaction, with increase of the number of atoms the central density of this condensate increases rapidly, leading to instability and collapse [7].

In the presence of vortex motion the region of largest density of the BEC with nonzero $L$ is pushed away from the central axial region and the atoms have more space to stabilize. The vortex state of the condensate in a cylindrical trap has the shape of a hollow cylinder with zero density on the axis of symmetry. Because of the larger spatial extension of such a condensate, it can accommodate a larger critical number of atoms before the density increases so high as to lead to 
collapse [9]. The higher the angular momentum $L$ in a BEC, the larger is the critical number of atoms. However, the increase of this critical number with increasing $L$ slows down as $L$ increases.

The present study is performed with direct numerical solution of the time-dependent GP equation with an axially symmetric trap. In the time evolution of the GP equation the radial and axial variables are dealt with in two independent steps. In each step the GP equation is solved by discretization with the Crank-Nicholson rule complemented by the known boundary conditions [25]. We find that this time-dependent approach leads to good convergence. There are several other iterative approaches to numerical solution of the timedependent and time-independent GP equations for axially symmetric $[4,9,10,26,27]$ as well as spherically symmetric [3] traps. Of the time-dependent methods, the approach of Refs. [10] uses alternate iterations in the radial and axial directions as in this study, whereas Ref. [26] does not give the details of the numerical method employed and Ref. [27] employs a completely different scheme, i.e., uses alternative iterations for the real and imaginary parts of the GP equation. However, Refs. $[9,10]$ do not provide enough details of the numerical scheme. Because of this a meaningful comparison of the present method with those of Refs. $[9,10,26,27]$ is not possible.

In Sec. II we describe the time-dependent form of the GP equation including the vortex states for attractive interaction. In Sec. III we describe in some detail the numerical method for solving the time-dependent GP equation. In Sec. IV we report the numerical results for the collapse of the BEC with the vortex quantum for attractive interaction and finally, in Sec. V, we give a summary of our investigation.

\section{NONLINEAR GROSS-PITAEVSKII EQUATION}

At zero temperature, the time-dependent Bose-Einstein condensate wave function $\Psi(\mathbf{r}, \tau)$ at position $\mathbf{r}$ and time $\tau$ may be described by the self-consistent mean-field nonlinear GP equation [8]. In the presence of a magnetic trap of cylindrical symmetry this equation is written as

$$
\left[-\frac{\hbar^{2}}{2 m} \nabla^{2}+V(\mathbf{r})+g N|\Psi(\mathbf{r}, \tau)|^{2}-i \hbar \frac{\partial}{\partial \tau}\right] \Psi(\mathbf{r}, \tau)=0 .
$$

Here $m$ is the mass of a single bosonic atom, $N$ the number of atoms in the condensate, $V(\mathbf{r})$ the attractive harmonic oscillator trap potential with cylindrical symmetry, and $g$ $=4 \pi \hbar^{2} \mathrm{a} / \mathrm{m}$ the strength of the interatomic interaction, with $a$ the atomic scattering length. A positive $a$ corresponds to a repulsive interaction and a negative $a$ to an attractive interaction. The normalization condition of the wave function is

$$
\int d \mathbf{r}|\Psi(\mathbf{r}, \tau)|^{2}=1
$$

The trap potential with cylindrical symmetry may be written as $V(\mathbf{r})=\frac{1}{2} m \omega^{2}\left(r^{2}+\lambda^{2} z^{2}\right)$ where $\omega$ is the angular frequency of the potential in the radial direction $r$ and $\lambda$ is the ratio of the axial to radial frequencies. We are using the cylindrical coordinate system $\mathbf{r} \equiv(r, \theta, z)$ and in the case of cylindrical symmetry the wave function is taken to be independent of $\theta$ in the absence of vortex states of the condensate:

$$
\Psi(\mathbf{r}, \tau)=\psi(r, z, \tau) .
$$

The GP equation with a cylindrically symmetric trap can easily accommodate quantized vortex states with rotational motion of the condensate around the $z$ axis without any added complication. In such a vortex the atoms flow with tangential velocity $L \hbar /(m r)$ such that each atom has quantized angular momentum $L \hbar$ along the $z$ axis. This corresponds to an angular dependence of

$$
\Psi(\mathbf{r}, \tau)=\psi(r, z, \tau) \exp (i L \theta)
$$

of the wave function, where $\exp (i L \theta)$ are the circular harmonics in two dimensions. Equation (2.3) is the zero angular momentum version of Eq. (2.4).

Substituting Eq. (2.4) into Eq. (2.1), one obtains the following GP equation in partial-wave form with quantized angular momentum $L$ along the $z$ axis:

$$
\begin{gathered}
{\left[-\frac{\hbar^{2}}{2 m}\left(\frac{1}{r} \frac{\partial}{\partial r} r \frac{\partial}{\partial r}+\frac{\partial^{2}}{\partial z^{2}}-\frac{L^{2}}{r^{2}}\right)+\frac{1}{2} m \omega^{2}\left(r^{2}+\lambda^{2} z^{2}\right)\right.} \\
\left.+g N|\psi(r, z, \tau)|^{2}-i \hbar \frac{\partial}{\partial \tau}\right] \psi(r, z, \tau)=0
\end{gathered}
$$

with $L=0,1,2, \ldots$. The nonzero values of $L$ correspond to vortex states. The $L^{2} / r^{2}$ term in Eq. (2.5) is the vortex contribution to the Hamiltonian of the GP equation. This is also the centrifugal barrier term in the partial-wave linear Schrödinger equation. The limitation to cylindrical symmetry reduces the GP equation in three space dimensions to a twodimensional partial differential equation. We shall study this equation numerically in this paper to understand the effect of the $L^{2} / r^{2}$ term on the collapse in the case of an attractive atomic interaction.

It is convenient to use dimensionless variables defined by $x=\sqrt{2} r / l, y=\sqrt{2} z / l, t=\tau \omega$, and

$$
\phi(x, y, t) \equiv \frac{\varphi(x, y, t)}{x}=\sqrt{\left(l^{3} / 2 \sqrt{2}\right)} \psi(r, z, \tau),
$$

where $l \equiv \sqrt{\hbar /(m \omega)}$. Although $\phi(x, y, t)$ is the dimensionless wave function, for calculational purpose we shall be using $\varphi(x, y, t)$ in the following. In terms of these variables Eq. (2.5) becomes

$$
\begin{gathered}
{\left[-\frac{\partial^{2}}{\partial x^{2}}+\frac{1}{x} \frac{\partial}{\partial x}-\frac{\partial^{2}}{\partial y^{2}}+\frac{L^{2}}{x^{2}}+\frac{1}{4}\left(x^{2}+\lambda^{2} y^{2}-\frac{4}{x^{2}}\right)\right.} \\
\left.+8 \sqrt{2} \pi n\left|\frac{\varphi(x, y, t)}{x}\right|^{2}-i \frac{\partial}{\partial t}\right] \varphi(x, y, t)=0,
\end{gathered}
$$

where $n=N a / l$. The reduced number of particles is defined as $|n|$. The normalization condition (2.2) of the wave function become 


$$
2 \pi \int_{0}^{\infty} d x \int_{-\infty}^{\infty} d y|\varphi(x, y, t)|^{2} x^{-1}=1
$$

However, physically it would be more interesting to define the reduced number of particles in terms of a geometrically averaged frequency $\omega_{0}=\lambda^{1 / 3} \omega$ and a length $l_{0}=\sqrt{\hbar / m \omega_{0}}$, so that a new reduced number $k(\lambda)$ is defined via [26]

$$
k(\lambda) \equiv \frac{N|a|}{l_{0}}=n \lambda^{1 / 6}
$$

We shall study this number in the present paper.

For a stationary solution the time dependence of the wave function is given by $\varphi(x, y, t)=\exp (-i \mu t) \varphi(x, y)$ where $\mu$ is the chemical potential of the condensate in units of $\hbar \omega$. If we use this form of the wave function in Eq. (2.7), we obtain the following stationary nonlinear time-independent GP equation $[8]$ :

$$
\begin{gathered}
{\left[-\frac{\partial^{2}}{\partial x^{2}}+\frac{1}{x} \frac{\partial}{\partial x}-\frac{\partial^{2}}{\partial y^{2}}+\frac{L^{2}}{x^{2}}+\frac{1}{4}\left(x^{2}+\lambda^{2} y^{2}-\frac{4}{x^{2}}\right)\right.} \\
\left.+8 \sqrt{2} \pi n\left|\frac{\varphi(x, y)}{x}\right|^{2}-\mu\right] \varphi(x, y)=0
\end{gathered}
$$

Equation (2.10) is the stationary version of the timedependent Eq. (2.7). However, Eq. (2.7) is equally useful for obtaining a stationary solution with trivial time dependence as well as for studying evolution processes with explicit time dependence, and we shall be directly solving Eq. (2.7) numerically in this paper.

Two interesting properties of the condensate wave function are the mean-square sizes in the radial and axial directions, defined, respectively, by

$$
\left\langle x^{2}\right\rangle=2 \pi \int_{0}^{\infty} d x \int_{-\infty}^{\infty} d y x|\varphi(x, y, t)|^{2}
$$

and

$$
\left\langle y^{2}\right\rangle=2 \pi \int_{0}^{\infty} d x \int_{-\infty}^{\infty} d y x^{-1} y^{2}|\varphi(x, y, t)|^{2} .
$$

\section{NUMERICAL METHOD}

To solve the time-independent GP equation we need the boundary conditions of the wave function as $x \rightarrow 0$ and $\infty$ and $|y| \rightarrow \infty$. For a confined condensate, for a sufficiently large $x$ and $|y|, \varphi(x, y)$ must vanish asymptotically. Hence the cubic nonlinear term can eventually be neglected in the GP equation for large $x$ and $|y|$ and Eq. (2.10) becomes

$$
\begin{aligned}
& {\left[-\frac{\partial^{2}}{\partial x^{2}}+\frac{1}{x} \frac{\partial}{\partial x}-\frac{\partial^{2}}{\partial y^{2}}+\frac{L^{2}}{x^{2}}\right.} \\
& \left.\quad+\frac{1}{4}\left(x^{2}+\lambda^{2} y^{2}-\frac{4}{x^{2}}\right)-\mu\right] \varphi(x, y)=0 .
\end{aligned}
$$

This is the equation for the free oscillator with cylindrical symmetry in partial-wave form. The wave function for a general state of this oscillator and the corresponding energy are given, respectively, by [28]

$$
\varphi(x, y)=\mathcal{N} x e^{-\left(x^{2}+\lambda y^{2}\right) / 4} F_{|L|, n_{x}}\left(\frac{x}{\sqrt{2}}\right) H_{n_{y}}\left(\frac{y \sqrt{\lambda}}{\sqrt{2}}\right)
$$

and

$$
\mu=\left(1+|L|+n_{x}\right)+\left(n_{y}+\frac{1}{2}\right) \lambda,
$$

with $L=0, \pm 1, \pm 2, \ldots, \quad n_{x}=0,2,4, \ldots, \quad$ and $n_{y}$ $=0,1,2, \ldots$. Here $H_{n_{y}}$ is the usual Hermite polynomial, $F_{|L|, n_{x}}$ is another polynomial defined recursively [28,29], and $\mathcal{N}$ is the normalization. The first few of these polynomials are $H_{0}(\xi)=1, \quad H_{1}(\xi)=2 \xi, \quad H_{2}(\xi)=\left(4 \xi^{2}-2\right), \quad H_{3}(\xi)$ $=\xi\left(8 \xi^{2}-12\right), \quad F_{0,0}(\xi)=1, \quad F_{1,0}(\xi)=\xi, \quad F_{2,0}(\xi)=\xi^{2}$, $F_{0,2}(\xi)=\left(1-\xi^{2}\right), \quad F_{3,0}(\xi)=\xi^{3}, \quad F_{1,2}(\xi)=\xi\left(\xi^{2}-4\right)$, etc. [29]. In this paper we shall be interested in angular momentum (vortex) excitation, as opposed to radial excitation via $n_{x}$ or axial excitation via $n_{y}$, of the following normalized ground state wave function for $n_{x}=n_{y}=0$ :

$$
\varphi(x, y)=\left(\frac{\lambda}{2^{2 L+3} \pi^{3}(|L| !)^{2}}\right)^{1 / 4} x^{1+|L|} e^{-\left(x^{2}+\lambda y^{2}\right) / 4},
$$

with energy

$$
\mu=1+|L|+\frac{1}{2} \lambda
$$

The solution (3.4) of Eq. (3.1) is a good starting point for the iterative method for solving the time-dependent GP equation (2.7) for small values of nonlinearity $n$ as in this paper. Alternatively, to solve the GP equation for large nonlinearity $n$, one may start with the Thomas-Fermi approximation for the wave function obtained by setting all the derivatives in the GP equation to zero [6], which is a good approximation for large nonlinearity.

Next we consider Eq. (2.7) as $x \rightarrow 0$. The nonlinear term approaches a constant in this limit because of the regularity of the wave function at $x=0$. Then one has the following condition:

$$
\varphi(0, y)=0
$$

as in the case of the harmonic oscillator wave function (3.4). Both the small- and large- $x$ behaviors of the wave function are necessary for a numerical solution of the time-dependent GP equation (2.7). The large- $x$ and large- $|y|$ behaviors of the wave function are given by Eq. (3.4), i.e.,

$$
\begin{aligned}
& \lim _{x \rightarrow \infty} \varphi(x, y) \rightarrow e^{-x^{2} / 4}, \\
& \lim _{|y| \rightarrow \infty} \varphi(x, y) \rightarrow e^{-\lambda y^{2} / 4} .
\end{aligned}
$$


A convenient way to solve Eq. (2.7) numerically is to discretize it in both space and time and reduce it to a set of algebraic equations, which can then be solved by using the known asymptotic boundary conditions. The method of solution using one space derivative is well under control $[3,25]$. The GP equation (2.7) can be written formally as

$$
i \frac{\partial}{\partial t} \varphi=H \varphi
$$

where $H$ is the time-independent quantity in the square brackets of Eq. (2.7). The integration in time is effected via the following semi-implicit Crank-Nicholson algorithm [25]:

$$
\frac{\varphi^{n+1}-\varphi^{n}}{-i \Delta}=\frac{1}{2} H\left(\varphi^{n+1}+\varphi^{n}\right)
$$

where $\Delta$ is the constant time step used to calculate the time derivative, $\varphi^{n}$ is the discretized wave function at time $t_{n}$ $=n \Delta$, and the space variables $x$ and $y$ are suppressed. The derivatives in the operator $H$ are discretized by the finite difference scheme [25]. The formal solution to Eq. (3.10) is given by

$$
\varphi^{n+1}=\frac{1-i \Delta H / 2}{1+i \Delta H / 2} \varphi^{n}
$$

so that if $\varphi^{n}$ is known at time $t_{n}$ one can find $\varphi^{n+1}$ at the next time step $t_{n+1}$. This procedure is used to solve the GP equation involving one space variable [3]. In that case after proper discretization in space using a finite difference scheme Eq. (3.11) becomes a tridiagonal set of equations in discrete space observables at time $t_{n+1}$, which is solved by the Gaussian elimination method and back substitution [25] using the known boundary conditions (3.6), (3.7), and (3.8). Unfortunately, a similar straightforward discretization of Eq. (2.7) in two space observables using a finite difference scheme in this case does not lead to a tridiagonal set of equations but rather to an unmanageable set of equations [25].

To circumvent this problem the full $H$ operator in this case is conveniently broken up into radial and axial components $H_{x}$ and $H_{y}$, respectively, where $H_{x}$ contains the terms dependent on $x$ and $H_{y}$ the terms dependent on $y$, with the nonlinear term $8 \sqrt{2} \pi n|\varphi(x, y) / x|^{2}$ involving both $x$ and $y$ contributing equally to both. Specifically, we take

$$
\begin{gathered}
H_{x}=-\frac{\partial^{2}}{\partial x^{2}}+\frac{1}{x} \frac{\partial}{\partial x}+\frac{L^{2}-1}{x^{2}}+\frac{x^{2}}{4}+4 \sqrt{2} \pi n\left|\frac{\varphi(x, y, t)}{x}\right|^{2}, \\
H_{y}=-\frac{\partial^{2}}{\partial y^{2}}+\frac{\lambda^{2} y^{2}}{4}+4 \sqrt{2} \pi n\left|\frac{\varphi(x, y, t)}{x}\right|^{2},
\end{gathered}
$$

with $H=H_{x}+H_{y}$. However, the numerical result of the present scheme is independent of the specific breakup.

The procedure is then to define the unknown wave function on a two-dimensional mesh in the $x-y$ plane. The time evolution is then performed in two steps. First the time evo- lution is effected using the operator $H_{x}$, setting $H_{y}=0$ along lines of constant $y$ with $i \partial \varphi / \partial t=H_{x} \varphi$. Next the time evolution is effected using the operator $H_{y}$, setting $H_{x}=0$ along lines of constant $x$ with $i \partial \varphi / \partial t=H_{y} \varphi$. This procedure is repeated alternately. This scheme is conveniently represented in terms of an auxiliary function $\varphi^{n+1 / 2}$ by

$$
\varphi^{n+1}=\frac{1-i \Delta H_{y} / 2}{1+i \Delta H_{y} / 2} \varphi^{n+1 / 2}, \quad \varphi^{n+1 / 2}=\frac{1-i \Delta H_{x} / 2}{1+i \Delta H_{x} / 2} \varphi^{n},
$$

so that

$$
\varphi^{n+1}=\frac{\left(1-i \Delta H_{y} / 2\right)}{\left(1+i \Delta H_{y} / 2\right)} \frac{\left(1-i \Delta H_{x} / 2\right)}{\left(1+i \Delta H_{x} / 2\right)} \varphi^{n}
$$

where $n=0,1,2, \ldots$ denotes the number of iterations. For a small time step $\Delta$, if we neglect terms quadratic in $\Delta$, Eq. (3.15) is equivalent to Eq. (3.11). Hence for numerical purposes we have been able to reduce the GP equation in two space dimensions $x$ and $y$ into a series of GP equations in one space variable, either $x$ or $y$. The GP equations in one space variable can be dealt with numerically in a standard fashion using Crank-Nicholson discretization and subsequent solution by the Gaussian elimination method. This scheme is stable independent of the time step employed.

The time-dependent GP equation (2.7) is solved by time iteration by mapping the solution on a two-dimensional grid of points $N_{x} \times N_{y}$ in $x$ and $y$. First Eq. (2.7) with $H_{x}$ is discretized using the following finite difference scheme along the $x$ direction within the semi-implicit Crank-Nicholson rule [25]:

$$
\begin{aligned}
\frac{i\left(\varphi_{j, p}^{n+1}-\varphi_{j, p}^{n}\right)}{\Delta}= & -\frac{1}{2 h^{2}}\left[\left(\varphi_{j+1, p}^{n+1}-2 \varphi_{j, p}^{n+1}+\varphi_{j-1, p}^{n+1}\right)\right. \\
& \left.+\left(\varphi_{j+1, p}^{n}-2 \varphi_{j, p}^{n}+\varphi_{j-1, p}^{n}\right)\right] \\
& +\frac{1}{4 x_{j} h}\left[\left(\varphi_{j+1, p}^{n+1}-\varphi_{n-1, p}^{n+1}\right)\right. \\
& \left.+\left(\varphi_{j+1, p}^{n}-\varphi_{j-1, p}^{n}\right)\right] \\
& +\left[\frac{x_{j}^{2}}{8}+\frac{L^{2}-1}{2 x_{j}^{2}}+2 \sqrt{2} \pi n \frac{\left|\varphi_{j, p}^{n}\right|^{2}}{x_{j}^{2}}\right] \\
& \times\left(\varphi_{j, p}^{n+1}+\varphi_{j, p}^{n}\right),
\end{aligned}
$$

where the discretized wave function $\varphi_{j, p}^{n} \equiv \varphi\left(x_{j}, y_{p}, t_{n}\right)$ refers to a fixed $y=y_{p}=p h, p=1,2, \ldots, N_{y}$ at different $x$ $=x_{j}=j h, j=1,2, \ldots, N_{x}$, and $h$ is the space step. This procedure results in a series of tridiagonal sets of equations (3.16) in $\varphi_{j+1, p}^{n+1}, \varphi_{j, p}^{n+1}$, and $\varphi_{j-1, p}^{n+1}$ at time $t_{n+1}$ for each $y_{p}$, which are solved by Gaussian elimination and back substitution [25] starting with the initial harmonic oscillator solution (3.4) at $t_{0}=0$ and $n=0$. Then Eq. (2.7) with $H_{y}$ is discretized using the following finite difference scheme along the $y$ direction: 


$$
\begin{aligned}
\frac{i\left(\varphi_{j, p}^{n+1}-\varphi_{j, p}^{n}\right)}{\Delta}= & -\frac{1}{2 h^{2}}\left[\left(\varphi_{j, p+1}^{n+1}-2 \varphi_{j, p}^{n+1}+\varphi_{j, p-1}^{n+1}\right)\right. \\
& \left.+\left(\varphi_{j, p+1}^{n}-2 \varphi_{j, p}^{n}+\varphi_{j, p-1}^{n}\right)\right] \\
& +\left[\frac{\lambda^{2} y_{p}^{2}}{8}+2 \sqrt{2} \pi n \frac{\left|\varphi_{j, p}^{n}\right|^{2}}{x_{j}^{2}}\right]\left(\varphi_{j, p}^{n+1}+\varphi_{j, p}^{n}\right),
\end{aligned}
$$

where now $\varphi_{j, p}^{n}$ refers to a fixed $x_{j}=j h$ for all $y_{p}=p h$. Using the solution obtained after $x$ iteration as input, the discretized tridiagonal equations (3.17) along the $y$ direction for constant $x$ are solved similarly. This two-step procedure corresponds to a full iteration of the GP equation and the resulting solution corresponds to time $t_{1}=\Delta$ and $n=1$. This scheme is repeated about 500 times to yield the final solution of the GP equation. The normalization condition (2.8) is preserved during time iteration due to the unitarity of the timeevolution operator. However, it is convenient to reenforce it numerically after each iteration in order to maintain a high level of precision. Also, the solution at each time step will satisfy the boundary conditions (3.6), (3.7), and (3.8). At each iteration the strength of the nonlinear term is increased by a small amount so that after about 500 time iterations the full strength is attained and the required solution of the GP equation obtained. The solution so obtained is iterated several times (between 20 and 50 times) until an equilibrated final result is obtained. This solution is the ground state of the condensate corresponding to the specific nonlinear constants $k$ and $L$.

We found the convergence of the two-step iteration scheme to be fast for small $|n|$. However, the final convergence of the scheme breaks down if $|n|$ is too large. For an attractive interaction there is no such problem as the GP equation does not sustain a large nonlinearity $|n|$. Typical values of the parameters used in this paper for discretization along the $x$ and $y$ directions are $N_{x}=400, N_{y}=800$, respectively, with $x_{\max }=8,|y|_{\max }=8$, and $\Delta=0.05$ for $\lambda>0.5$. For smaller $\lambda(<0.5)$ the wave function extends further along the $y$ axis and larger $\left|y_{\max }\right|$ and $N_{y}$ are employed to obtain a converged result. The above choice of parameters corresponds to a typical space step of $h=0.02$ along both radial and axial directions. These parameters were obtained after some experimentation and are found to lead to good convergence.

As the time dependence of the stationary states is trivial$\varphi(x, y, t)=\varphi(x, y) \exp (-i \mu t)$ - the chemical potential $\mu$ can be obtained from the propagation of the converged ground state solution at two times, e.g., $\varphi\left(x, y, t_{n}\right)$ and $\varphi\left(x, y, t_{n+n^{\prime}}\right)$. From the numerically obtained ratio $\varphi\left(x, y, t_{n}\right) / \varphi\left(x, y, t_{n+n^{\prime}}\right)=\exp \left(i \mu n^{\prime} \Delta\right), \mu$ can be obtained as the time step $\Delta$ is known. In the calculation of $\mu$ an average over relatively large values of $n^{\prime}$ leads to a stable result.

\section{NUMERICAL RESULTS}

Using the numerical method described in Sec. III we present results in this section for the numerical solution of the time-dependent GP equation for an attractive interatomic interaction, with special attention to the collapse of the condensate. To assure that we are on the correct track using the present program we first solved the GP equation for the spherically symmetric case with $\lambda=1$ and $L=0$, and compared our results with the calculation of Ref. [30]. As an additional check we also solved the GP equation in two space dimensions with $\lambda=0$ and without the $d^{2} / d y^{2}$ term in Eq. (2.7) and compared with the calculation of Ref. [31]. In both cases the present calculation agrees with these previous ones.

Before describing the results for nonzero $L$ we first compare the present results for $L=0$ with those of Ref. [26] for a cylindrically symmetric trap. For the spherically symmetric case $\lambda=1$, and the critical number $k_{c}(\lambda)$ of Eq. (2.9) for collapse is found to be 0.575 in agreement with Refs. $[6,26,30]$. In a recent experiment using $\lambda=0.3919$, the critical reduced number for collapse for an attractive condensate of ${ }^{85} \mathrm{Rb}$ atoms formed using a Feshbach resonance was found to be $k_{c}=0.459 \pm 0.012 \pm 0.054$ [32]. In their calculation Gammal et al. [26] obtained $k_{c}=0.550$ for $\lambda=0.3919$. In the present calculation we obtain $k_{c}=0.553$, in excellent agreement with Ref. [26], using an entirely different numerical routine. However, the disagreement with the experimental result [32] remains. We also calculated the critical number $k_{c}(\lambda)$ for some other values of $\lambda$. For $\lambda=5,2$, and 0.2 we obtain $k_{c}=0.50,0.56$, and 0.52 , respectively, compared to $0.498,0.561$, and 0.509 obtained in Ref. [26]. The small difference between the results of the two calculations seems to be a consequence of numerical error. Also, as in Ref. [26] we note that for $\lambda$ not so different from unity $(5>\lambda>0.2)$ the critical reduced number for collapse $k_{c}(\lambda)$ satisfies $k_{c}(\lambda) \approx k_{c}(1 / \lambda)$, and attains a maximum at $\lambda=1$ corresponding to the spherically symmetric situation. However, this symmetry is broken for large values of $\lambda$, e.g., for $\lambda$ $>5$, where we have $k_{c}(\lambda)<k_{c}(1 / \lambda)$. Moreover, we find in the following that this symmetry is also broken for nonzero $L$, where, however, for $\lambda>1, k_{c}(\lambda)>k_{c}(1 / \lambda)$.

Next we comment on the discrepancy between the experimental critical number of atoms for collapse for an attractive BEC of ${ }^{85} \mathrm{Rb}$ atoms formed using a Feshbach resonance [32] on one hand and the theoretical results of Ref. [26] and the present calculation on the other hand. In view of the success of the mean-field GP equation in explaining many stationary results and time-evolution phenomena of the attractive BEC of ${ }^{7} \mathrm{Li}$ atoms with an almost spherical trap [2,7], it seems that this description is perfectly appropriate for attractive condensates. Hence, we do not believe that a relatively small deviation from spherical symmetry as in the experiment of Ref. [32] would invalidate the applicability of the GP equation to an attractive condensate. Whether the inclusion of higher order interaction terms in the mean-field GP equation could account for the observed data [26] yet remains to be established. To resolve the discrepancy we advocate further experimental study of collapse for attractive condensates after changing the trap symmetry $(\lambda)$.

After the above preliminary comparative study, we present results for the numerical solution of the GP equation (2.7) for nonzero $L=0,1,2, \ldots, 8$ and $\lambda=\sqrt{8}$ and $1 / \sqrt{8}$ for 


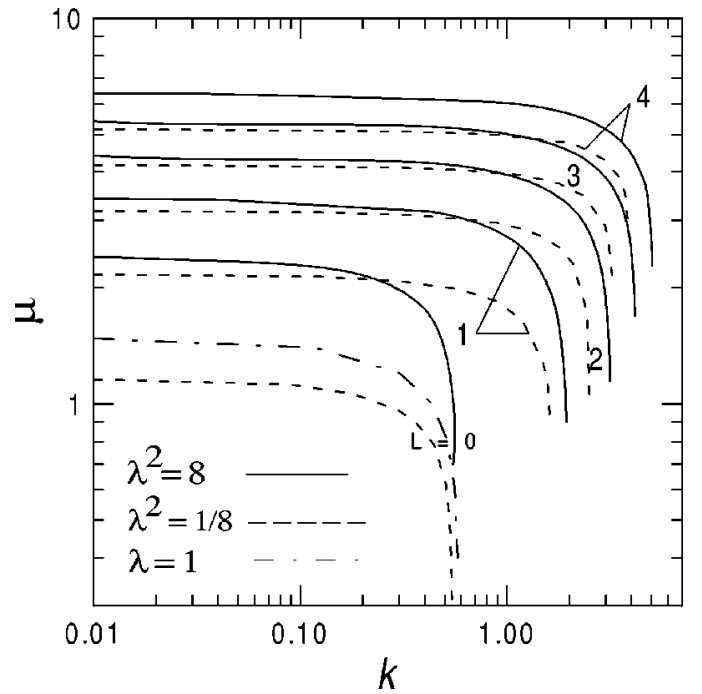

FIG. 1. Chemical potential $\mu$ vs reduced number $k$ for different $\lambda$ and $L$. The curves are labeled by their $L$ values.

different $k(\lambda)$. We recall that $\lambda=\sqrt{8}$ corresponds to the experiment of Ensher et al. [1] for the BEC of ${ }^{87} \mathrm{Rb}$ atoms. These two possibilities for $\lambda$ correspond to axial compression $(\lambda>1)$ and elongation $(\lambda<1)$ of the condensate. For each $L$ we increase $k$ from 0 and calculate the chemical potential $\mu$. With increase of $k$ the wave function becomes more and more localized in space, and beyond a certain value of $k$ the density at the peak of the wave function diverges and no stable normalizable solution of the GP equation with a well defined $\mu$ can be obtained.

In Fig. 1 we plot $\mu$ vs $k(\lambda)$ for $\lambda=\sqrt{8}$ and $1 / \sqrt{8}$ for different $L$. We also exhibit the result for the spherically symmetric case $\lambda=1(L=0)$ for comparison. The curves are plotted for all allowed values of $k$ for the ground state in each case. The curves go up to a maximum critical value $k_{c}$ of $k$ which defines the critical number $N_{c}$ of atoms in that particular case via $k_{c}=N_{c}|a| / l_{0}$. We find that (i) $k_{c}$ for a particular $\lambda$ increases with increasing $L$ and (ii) $k_{c}$ for a particu-

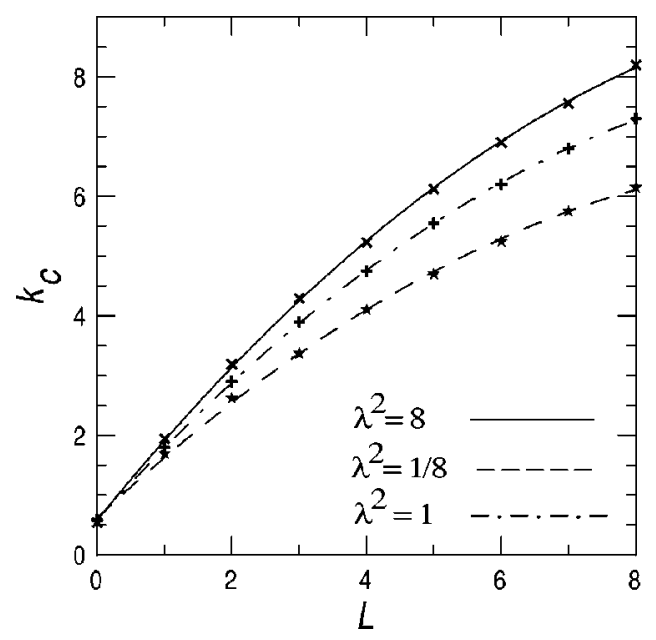

FIG. 2. Critical reduced number $k_{c}$ vs $L$ for $\lambda=\sqrt{8}$ (full line with $\times$ ), 1 (dash-dotted line with + ), and $1 / \sqrt{8}$ (dashed line with $\star)$. The lines are polynomial fits to the points.
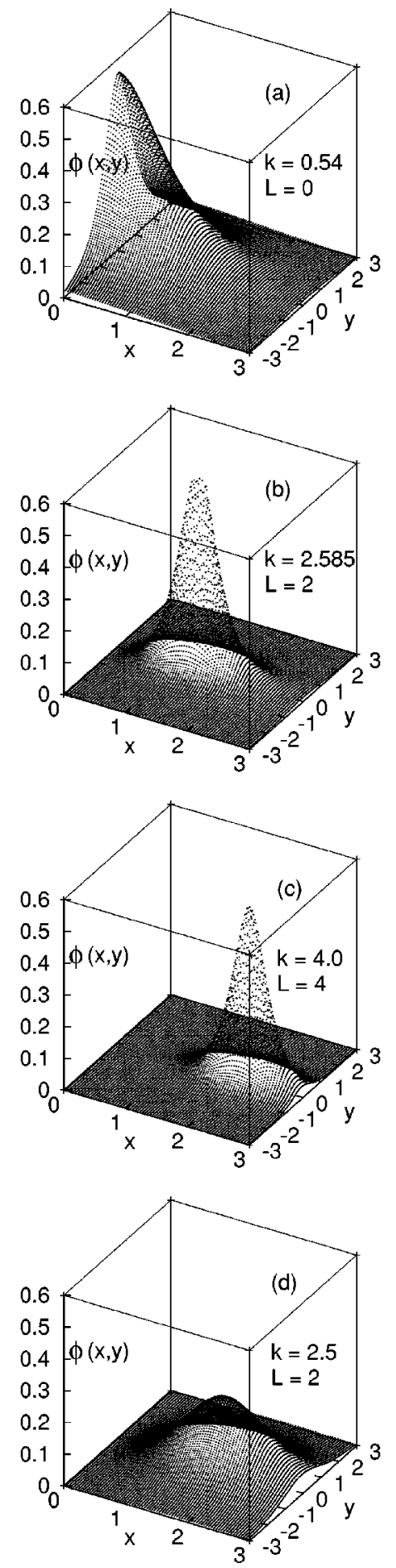

FIG. 3. The wave function $|\phi(x, y)| \equiv|\varphi(x, y) / x|$ vs $x$ and $y$ for $\lambda=1 / \sqrt{8}$ and for (a) $L=0, k=0.54$, (b) $L=2, k=2.58$, (c) $L=4$, $k=4.00$, and (d) $L=2, k=2.50$.

lar nonzero $L$ increases as $\lambda$ increases from $1 / \sqrt{8}$ to $\sqrt{8}$, which demonstrates the breakdown of the numerically noted symmetry $k_{c}(\lambda) \approx k_{c}(1 / \lambda)$ for $L=0$. To demonstrate these two effects in an explicit fashion we plot in Fig. $2 k_{c}$ vs $L$ for $\lambda=\sqrt{8}, 1$, and $1 / \sqrt{8}$. The three curves intersect at approximately $L=0$, which demonstrates that $k_{c}(\lambda=\sqrt{8}) \approx k_{c}(\lambda$ $=1 / \sqrt{8})<k_{c}(\lambda=1)$ for $L=0$, with $k_{c}(\lambda=\sqrt{8})=0.54, k_{c}(\lambda$ 

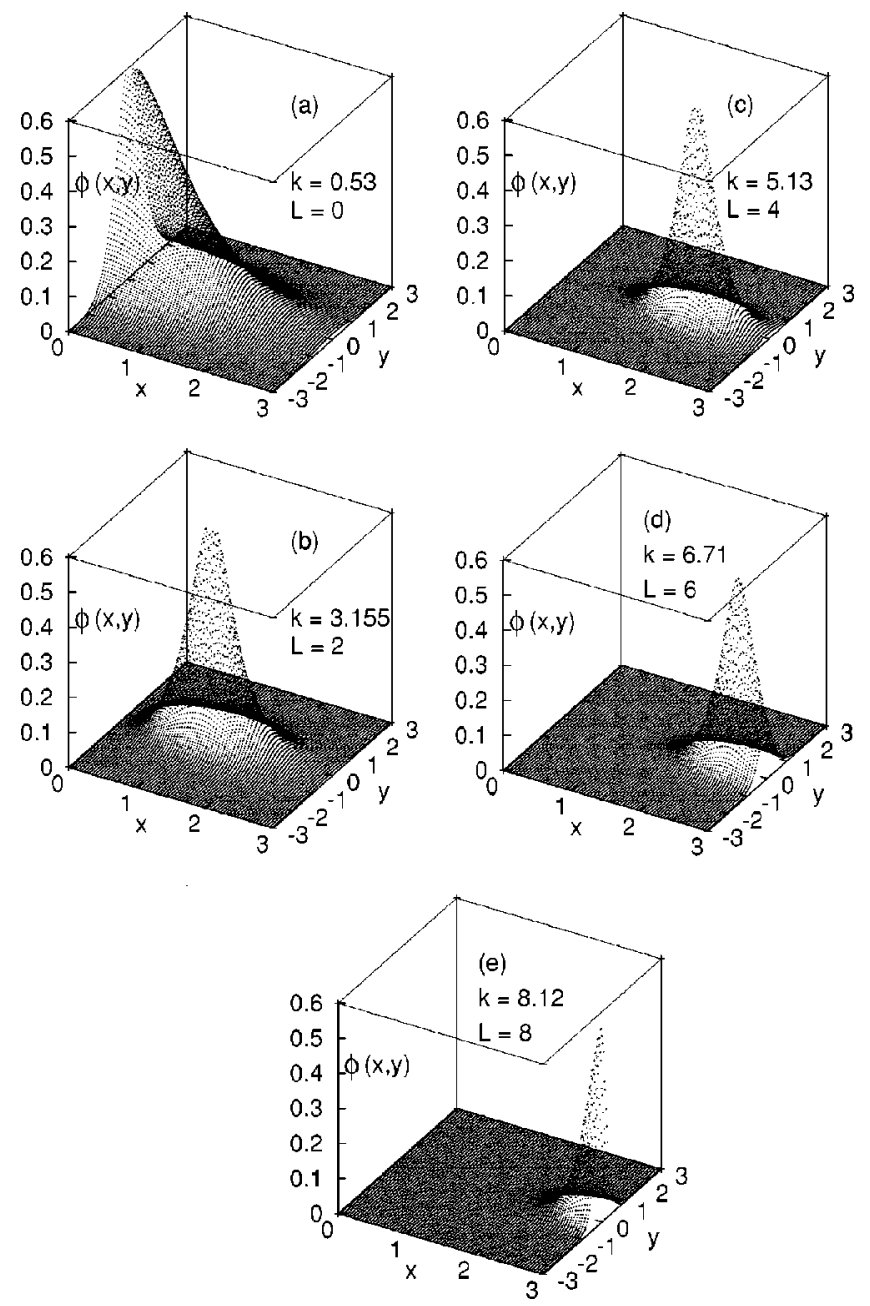

FIG. 4. Same as Fig. 3 for $\lambda=\sqrt{8}$ and for (a) $L=0, k=0.53$, (b) $L=2, k=3.15$, (c) $L=4, k=5.13$, (d) $L=6, k=6.71$, and (e) $L$ $=8, k=8.12$.

$=1 / \sqrt{8})=0.55$, and $k_{c}(\lambda=1)=0.575$. However, this symmetry is broken for nonzero $L$ where $k_{c}(\lambda=\sqrt{8})>k_{c}(\lambda$ $=1)>k_{c}(\lambda=1 / \sqrt{8})$. The critical number $k_{c}(\lambda)$ increases with increasing $L$ for all $\lambda$, and we see from Fig. 2 that this rate of increase slows down as $L$ increases.

In Figs. 3 and 4 we plot the wave function $|\phi(x, y)|$ $\equiv|\varphi(x, y) / x|$ in dimensionless variables of Eq. (2.6). In Figs. $3(\mathrm{a})-3(\mathrm{c})$ we show the wave function for $\lambda=1 / \sqrt{8}$ and $L$ $=0,2$, and 4 , respectively, where the parameter $k$ is chosen to be very close to the critical value $k_{c}$ for collapse. The nature of the wave function is qualitatively different for zero and nonzero $L$. For $L=0$ the wave function is peaked on the $y$ axis; whereas for nonzero $L$ it is zero on the $y$ axis and peaked at some finite $x$. In all cases the peak is sharp and the density of atoms is very large at the peak. The BEC collapses with a slight increase in the parameter $k$. For smaller $k$ the wave function has a much broader maximum. When $k$ approaches $k_{c}$ a sharp maximum of the wave function appears very rapidly. To illustrate this in Fig. 3(d) we plot the $L=2$ wave function for $k=2.5$. If we compare this with the wave function of Fig. 3(b) for $L=2$ and $k=2.58 \approx k_{c}$, the change in the shape is explicit.
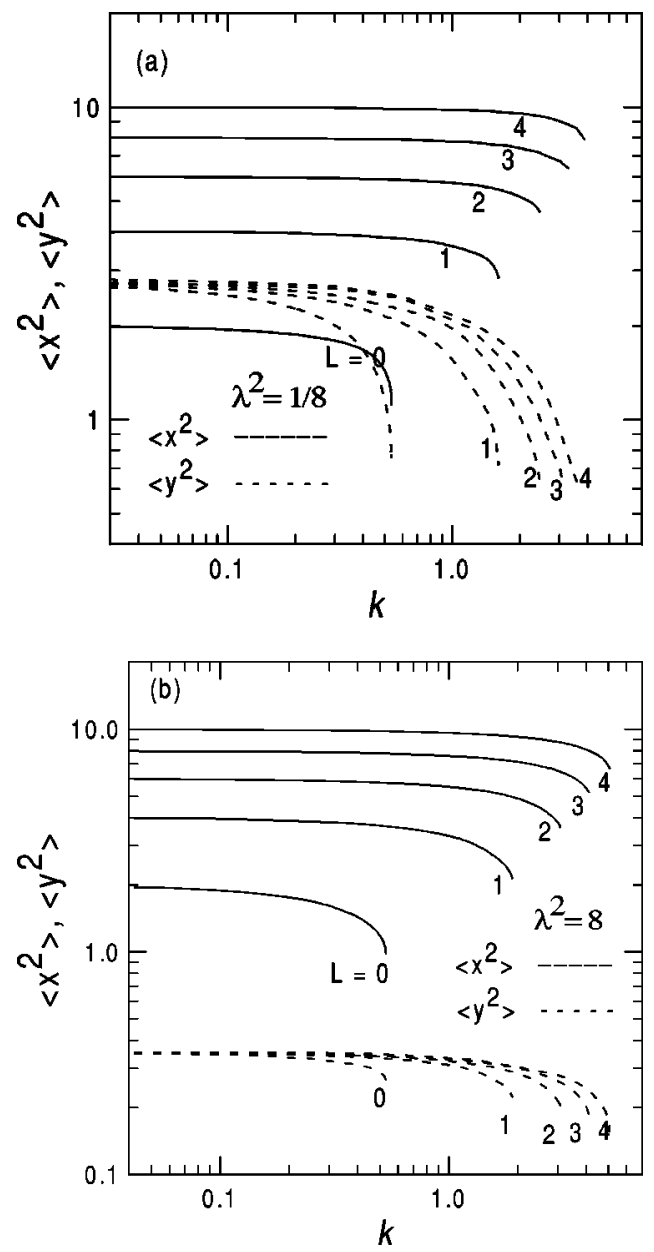

FIG. 5. Mean-square sizes $\left\langle x^{2}\right\rangle$ (full lines) and $\left\langle y^{2}\right\rangle$ (dashed lines) vs reduced number $k$ for (a) $\lambda=1 / \sqrt{8}$ and (b) $\lambda=\sqrt{8}$.

In Figs. 4(a)-4(e) we plot the wave function for $\lambda=\sqrt{8}$ and for $L=0,2,4,6$, and 8 , respectively, for $k \approx k_{c}$. If we compare Figs. 3 and 4 for the same $L$ we find that for $\lambda$ $=1 / \sqrt{8}$ the wave functions extend over a larger region along the $y$ axis compared to those for $\lambda=\sqrt{8}$. This is apparent if we compare Fig. 3(a) with Fig. 4(a), and is expected as $\lambda$ $=\sqrt{8}$ corresponds to a stronger harmonic oscillator potential in the $y$ direction responsible for axial compression. From Figs. 3 and 4 we find that for both $\lambda$ values the peak in the wave function moves further away from the $y$ axis as $L$ increases.

To understand some aspects of the variation of $k_{c}$ with $L$ and $\lambda$ exhibited in Fig. 2, we plot in Figs. 5(a) and 5(b) the mean-square sizes $\left\langle x^{2}\right\rangle$ and $\left\langle y^{2}\right\rangle$ vs $k$ for different $L$ and for $\lambda=1 / \sqrt{8}$ and $\sqrt{8}$, respectively. The results for vortex states $(L>0)$ in the spherically symmetric case with $\lambda=1$ remain between those for $\lambda=1 / \sqrt{8}$ and $\sqrt{8}$ and are not explicitly shown here. For nonzero $L$ the system acquires a positive rotational energy $L^{2} / x^{2}$ which allows it to move away from the axial direction $y$. For $L=0$ the region of highest density is the $y$ axis. For $L \neq 0$ the density is zero on the $y$ axis and has a maximum at some finite $x$. Consequently, the condensate has the shape of a hollow cylinder. Because of vortex motion the condensate swells and has more space to stabi- 
lize. Hence for $L>0$ the density does not go to an unstable level with the same number of atoms as for $L=0$, and $k_{c}$ increases with increasing $L$ for all $\lambda$. However, for all $L$ and $\lambda$, with an increase of nonlinearity $k$ (or $n$ ) in the GP equation (2.7), the attractive nonlinear interaction term takes control and eventually the mean-square sizes $\left\langle x^{2}\right\rangle$ and $\left\langle y^{2}\right\rangle$ are reduced as can be seen from Fig. 5. This eventual shrinking in size with increase of the number of atoms for all $L$ and $\lambda$ together with the outward push due to vortex motion for nonzero $L$ takes the density of the BEC at the maximum of the wave function to an unstable high level at some critical value $k_{c}$ of $k$, leading to collapse.

Although, for a fixed $\lambda, k_{c}$ increases with increasing $L$, the rate of increase slows down for large $L$. As $k$ (or $n$ ) increases sufficiently for large $L(>8)$, the nonlinear term containing $n$ becomes the deciding factor in the GP equation and the $L^{2} / x^{2}$ term starts to play a secondary role. Consequently, the increase in the critical number $k_{c}$ with increasing $L$ slows down as $L$ increases and the number $k_{c}$ tends to saturate as can be seen clearly in Fig. 2 . In all cases $(\lambda$ $=\sqrt{8}, 1$, and $1 / \sqrt{8})$ this tendency to saturation is visible beyond $L=4$.

\section{SUMMARY}

In this paper we present a numerical study of the timedependent Gross-Pitaevskii equation under the action of a harmonic oscillator trap with cylindrical symmetry with attractive interparticle interaction to obtain insight into the collapse of vortex states of BEC's. The time-dependent GP equation is solved iteratively by discretization using a twostep Crank-Nicholson scheme. We obtain the boundary conditions (3.6), (3.7), and (3.8) of the solution of the dimensionless GP equation (2.7) and use them for its solution. The solution procedure is applicable for both attractive and repulsive atomic interactions as well as for both stationary and time-evolution problems. It is expected that numerical difficulty will appear for large nonlinearity or large values of the reduced number of particles $k$ and for large vortex quantum number $L$. For medium nonlinearity, as in this paper, the accuracy of the time-independent method can be increased by reducing the space and time steps used in discretization.

The ground state wave function for each $L$ is found to be sharply peaked for attractive interatomic interaction with the parameters set close to those for collapse. In the case of an attractive interaction, the mean-square sizes $\left\langle x^{2}\right\rangle$ and $\left\langle y^{2}\right\rangle$ decrease as the number of particles in the condensate increases toward the critical number for collapse. Consequently, the density increases rapidly, signaling the onset of collapse beyond a critical reduced number $k_{c}$.

The presence of the quantized vortex states increases the stability of the BEC with attractive interaction. The critical number $k_{c}(\lambda)$ for $L=0$ is largest in the spherically symmetric case $\lambda=1$. For vortex states $(L \neq 0), k_{c}(\lambda)$ increases with increasing $\lambda$. As the vortex quantum number $L$ increases, $k_{c}$ also increases. However, in the present calculation a tendency to saturation in the value of $k_{c}$ is noted with increasing $L$. As the parameter $n$ or $k$ in the GP equation increases, the nonlinear term starts to play the dominating role in the GP equation compared to the angular momentum term $L^{2} / x^{2}$. Once this happens, the rate of increase of $k_{c}$ with $L$ slows down, and it is not unlikely that the critical number will attain a limiting maximum value for a larger $L(>8)$ than those considered in this paper. This and other investigations into the collapse of vortex states are welcome in the future.

\section{ACKNOWLEDGMENTS}

This work was supported in part by the Conselho Nacional de Desenvolvimento Científico e Tecnológico and by Fundação de Amparo à Pesquisa do Estado de São Paulo of Brazil.
[1] M. H. Anderson, J. R. Ensher, M. R. Matthews, C. E. Wieman, and E. A. Cornell, Science 269, 198 (1995); J. R. Ensher, D. S. Jin, M. R. Matthews, C. E. Wieman, and E. A. Cornell, Phys. Rev. Lett. 77, 4984 (1996); K. B. Dadic, M. O. Mewes, M. R. Andrews, N. J. van Druten, D. S. Durfee, D. M. Kurn, and W. Ketterle, ibid. 75, 3969 (1995); D. G. Fried, T. C. Killian, L. Willmann, D. Landhuis, S. C. Moss, D. Kleppner, and T. J. Greytak, ibid. 81, 3811 (1998); F. Pereira Dos Santos, J. Léonard, Junmin Wang, C. J. Barrelet, F. Perales, E. Rasel, C. S. Unnikrishnan, M. Leduc, and C. Cohen-Tannoudji, ibid. 86, 3459 (2001).

[2] C. C. Bradley, C. A. Sackett, J. J. Tollett, and R. G. Hulet, Phys. Rev. Lett. 75, 1687 (1995); C. A. Sackett, H. T. C. Stoof, and R. G. Hulet, ibid. 80, 2031 (1998); C. C. Bradley, C. A. Sackett, and R. G. Hulet, ibid. 78, 985 (1997); J. M. Gerton, D. Strekalov, I. Prodan, and R. G. Hulet, Nature (London) 408, 692 (2000).

[3] M. Edwards and K. Burnett, Phys. Rev. A 51, 1382 (1995); P. A. Ruprecht, M. J. Holland, K. Burnett, and M. Edwards, ibid.
51, 4704 (1995); S. K. Adhikari, Phys. Rev. E 63, 056704 (2001).

[4] B. I. Schneider and D. L. Feder, Phys. Rev. A 59, 2232 (1999).

[5] M. Edwards, P. A. Ruprecht, K. Burnett, R. J. Dodd, and C. W. Clark, Phys. Rev. Lett. 77, 1671 (1996); M. J. Holland, D. S. Jin, M. L. Chiofalo, and J. Cooper, ibid. 78, 3801 (1997).

[6] F. Dalfovo, S. Giorgini, L. P. Pitaevskii, and S. Stringari, Rev. Mod. Phys. 71, 463 (1999).

[7] Yu. Kagan, A. E. Muryshev, and G. V. Shlyapnikov, Phys. Rev. Lett. 81, 933 (1998); M. Ueda and A. J. Leggett, ibid. 80, 1576 (1998); V. S. Filho, A. Gammal, T. Frederico, and L. Tomio, Phys. Rev. A 62, 033605 (2000); M. Ueda and K. Huang, ibid. 61, 043601 (1999); F. Kh. Abdullaev, A. Gammal, L. Tomio, and T. Frederico, ibid. 63, 043604 (2001); R. J. Dodd, M. Edwards, C. J. Williams, C. W. Clark, M. J. Holland, P. A. Ruprecht, and K. Burnett, ibid. 54, 661 (1996); M. Houbiers and H. T. C. Stoof, ibid. 54, 5055 (1996); A. Eleftheriou and K. Huang, ibid. 61, 043601 (2000); L. Bergé, T. J. Alexander, and Y. S. Kivshar, ibid. 62, 023607 (2000); S. K. Adhikari, 
Phys. Lett. A 281, 265 (2001); Phys. Rev. A 63, 043611 (2001); J. Phys. B 34, 4231 (2001); A. Gammal, T. Frederico, L. Tomio, and F. Kh. Abdullaev, Phys. Lett. A 267, 305 (2000); M. Wadati and T. Tsurumi, ibid. 247, 287 (1998).

[8] E. P. Gross, Nuovo Cimento 20, 454 (1961); L. P. Pitaevskii, Zh. Eksp. Teor. Fiz. 40, 646 (1961) [Sov. Phys. JETP 13, 451 (1961)].

[9] F. Dalfovo and S. Stringari, Phys. Rev. A 53, 2477 (1996).

[10] M. Holland and J. Cooper, Phys. Rev. A 53, R1954 (1996); F. Dalfovo and M. Modugno, ibid. 61, 023605 (2000).

[11] B. Jackson, J. F. McCann, and C. S. Adams, Phys. Rev. A 61, 013604 (1999).

[12] D. L. Feder, C. W. Clark, and B. I. Schneider, Phys. Rev. Lett. 82, 4956 (1999).

[13] G. M. Kavoulakis, B. Mottelson, and C. J. Pethick, Phys. Rev. A 62, 063605 (2000).

[14] J. J. García-Ripoll and V. M. Pérez-García, Phys. Rev. Lett. 84, 4264 (2000); V. M. Pérez-García and J. J. García-Ripoll, Phys. Rev. A 62, 033601 (2000).

[15] F. Dalfovo and S. Stringari, Phys. Rev. A 63, 011601 (2001); A. Recati, F. Zambelli, and S. Stringari, Phys. Rev. Lett. 86, 377 (2001); D. Guery-Odelin and S. Stringari, ibid. 83, 4452 (1999); S. Stringari, ibid. 82, 4371 (1999).

[16] J. E. Williams and M. J. Holland, Nature (London) 401, 568 (1999).

[17] D. A. Butts and D. S. Rokshar, Nature (London) 397, 327 (1999).

[18] K. W. Madison, F. Chevy, W. Wohlleben, and J. Dalibard, Phys. Rev. Lett. 84, 806 (2000); K. W. Madison, F. Chevy, V. Bretin, and J. Dalibard, ibid. 86, 4443 (2001).
[19] M. R. Matthews, B. P. Anderson, P. C. Haljan, D. S. Hall, C. E. Wieman, and E. A. Cornell, Phys. Rev. Lett. 83, 2498 (1999).

[20] R. J. Marshall, G. H. C. New, K. Burnett, and S. Choi, Phys. Rev. A 59, 2085 (1999).

[21] R. Dum, J. I. Cirac, M. Lewenstein, and P. Zoller, Phys. Rev. Lett. 80, 2972 (1998).

[22] A. A. Svidzinsky and A. L. Fetter, Phys. Rev. A 62, 063617 (2000).

[23] S. L. Cornish, N. R. Claussen, J. L. Roberts, E. A. Cornell, and C. E. Wieman, Phys. Rev. Lett. 85, 1795 (2000).

[24] E. Timmermans, P. Tommasini, M. Hussein, and A. Kerman, Phys. Rep. 315, 199 (1999).

[25] S. E. Koonin and D. C. Meredith, Computational Physics Fortran Version (Addison-Wesley, Reading, MA, 1990), pp. 169180.

[26] A. Gammal, T. Frederico, and L. Tomio, Phys. Rev. A 64, 055602 (2001).

[27] M. M. Cerimele, M. L. Chiofalo, F. Pistella, S. Succi, and M. P. Tosi, Phys. Rev. E 62, 1382 (2000).

[28] L. Pauling and E. B. Wilson, Introduction to Quantum Mechanics (McGraw-Hill, New York, 1935), pp. 105-111.

[29] W. V. Houston and G. C. Phillips, Principles of Quantum Mechanics (North-Holland, Amsterdam, 1973), pp. 70-73.

[30] A. Gammal, T. Frederico, and L. Tomio, Phys. Rev. E 60, 2421 (1999).

[31] S. K. Adhikari, Phys. Lett. A 265, 91 (2000); Phys. Rev. E 62, 2937 (2000)

[32] J. L. Roberts, N. R. Claussen, S. L. Cornish, E. A. Donley, E. A. Cornell, and C. E. Wieman, Phys. Rev. Lett. 86, 4211 (2001). 